Aim of the study: To investigate the impact of hyperthermic intraperitoneal chemotherapy (HIPEC) on the clinical and oncological outcomes and quality of life $(\mathrm{QOL})$ of patients with peritoneal carcinomatosis (PC).

Material and methods: The study involved 304 patients with PC of different origin, who were divided into 2 groups: Group I - cytoreductive surgery (CRS) + adjuvant chemotherapy (ACT) - 247 patients; Group II - CRS + HIPEC + ACT - 57 patients. Intraoperative characteristics and postoperative complications were compared. Patients' QOL was assessed at all phases of treatment using the international scales the Short Form-36 Health Survey (SF-36) and European Organisation for Research and Treatment of Cancer Quality of Life Questionnaire for Breast Cancer Core 30 (EORTC QLQ-C30). Results: No statistically significant differences were observed between the 2 groups comparing the average blood loss and the total rate of postoperative complications, although the rates of hyperthermia and acute renal failure in the early postoperative period were higher in the HIPEC group. The use of HIPEC significantly contributed to the worse restoration of intestinal function in the postoperative period and to prolonged hospital stay. Assessment of the QOL of patients in Group II using SF-36 showed no significant difference between the physical and psychological components of health compared with the control group. The analysis of EORTC data showed a significant deterioration in the QOL of patients in Group II due to increased scales of pain, nausea and vomiting, and constipation in the early postoperative period. No difference in $\mathrm{QOL}$ was observed in the subsequent phases of treatment and after its completion. Overall survival and disease-free survival of patients with ovarian cancer who underwent HIPEC were significantly better compared with CRS + ACT alone.

Conclusions: The proposed HIPEC technique has demonstrated its clinical safety in the treatment of PC, no long-term negative impact on the $\mathrm{QOL}$ of patients, and better oncological results for ovarian cancer.

Key words: peritoneal carcinomatosis, hyperthermic intraperitoneal chemotherapy, cytoreductive surgery, quality of life.

Contemp Oncol (Pozn) 2021; 25 (4): 270-278 DOI: https://doi.org/10.5114/wo.2021.112038

\section{The impact of hyperthermic intraperitoneal chemotherapy and cytoreductive surgery on clinical outcomes and quality of life of patients with peritoneal carcinomatosis}

\author{
Oleksandr Vadymovych Bondar, Serhii Hennadiiovych Chetverikov, \\ Viacheslav Yevheniiovych Maksymovskyi, Dmytro Vadymovych \\ Atanasov, Valeriia Volodymyrivna Chetverikova-Ovchynnyk, \\ Mykhailo Serhiiovych Chetverikov
}

Odessa National Medical University, Ukraine

\section{Introduction}

Peritoneal carcinomatosis (PC) is a metastatic deposition across the peritoneum with a malignant tumor mass of various primary origin. Peritoneal carcinomatosis remains a problem of current interest for timely diagnosis and treatment.

The term 'peritoneal carcinomatosis' was first used by Sampson in 1931 to describe metastatic implants of ovarian cancer across the peritoneal cavity [1].

Over the past decades, PC was regarded to be hopeless as the terminal stage of neoplastic disease due to its negative prognosis. Surgical treatment was not recommended. Palliative systemic chemotherapy has remained the only way to prolong life for patients with PC. The median survival of those patients with $P C$ who receive the best supportive care usually does not exceed 3-14 months, depending on the primary origin [2-5].

In most cases, the primary sites of peritoneal cancer implants are malignant gynecological and gastrointestinal tumors. Malignant peritoneal mesothelioma has a similar mechanism of dissemination. The incidence of synchronous PC in patients with colorectal cancer is 5-15\% [2-4], for gastric cancer $14-20 \%[2,6]$, for ovarian cancer $70-80 \%[2,7]$.

The pattern of implantation metastasis is explained by the primary tumor's biology and the histological structure of the peritoneum. Both these factors allow us to regard the process as locoregional (S. Paget's "seed" and "soil" cancer theory) [8]. Implantation theory of PC supports the effectiveness of cytoreductive treatment, which increases the patients' quality of life (QOL) and overall survival.

The paradigm shift in the therapy of PC of different primary origins, which was observed in recent decades, has been accompanied by the introduction of new aggressive methods of treatment. The concept of cytoreductive surgery (CRS) was proposed by Paul H. Sugarbaker in 1985. It is based on the principle of maximum removal of the macroscopically visible tumor mass from the abdominal cavity in order to achieve a minimum microscopic level of the residual intraperitoneal pool of tumor cells which helps to achieve the best effect of chemotherapeutic drugs.

Intraperitoneal administration of cytostatic antitumor drugs in malignant pathologies of the abdominal cavity dates back to 1955, when Jaaback et al. published studies of the intraperitoneal infusion of chloroethylamines 
in ascites of malignant etiology [9]. The combination of optimal CRS and hyperthermic intraperitoneal chemotherapy (HIPEC) is currently proven as pathogenetically reasonable for the treatment of PC of different primary origin, especially when there are no hematogenous metastases.

Hyperthermia increases the permeability of chemotherapy drugs through the peritoneum and increases the sensitivity of malignant cells to cytostatic drugs due to impaired DNA repair. Hyperthermia also induces apoptosis, inhibits angiogenesis, and has a direct cytotoxic effect by promoting protein denaturation [10]. An increase of temperature leads to increased accumulation of cisplatin in platinum-resistant cell lines. Some studies have linked the effect of hyperthermia in HIPEC to the inhibition of superoxide dismutase, a potent antioxidant enzyme that helps to overcome the oxidative stressors of chemotherapeutic drugs in tumor cells [11]. The effect of hyperthermic chemoperfusion according to Bhatt et al. is observed at depths up to 3-5 $\mathrm{mm}$ [12]. Intraperitoneal chemotherapy in the condition of hyperthermia has a better ability to penetrate into the retroperitoneal space, where tumor deposits may also be located. The formation of adhesions after CRS prevents the equable distribution of drugs that are introduced intraperitoneally. It may be neutralized by hyperthermia, which increases the permeability of the peritoneum [13]. Hyperthermic intraperitoneal chemotherapy makes it possible to achieve a high concentration of chemotherapeutic drugs in the abdominal cavity with minimal systemic action and to cross the blood-peritoneal barrier. Peritoneal perfusion also provides the mechanical washout of tumor cells from the abdominal cavity. Therefore, the effect of HIPEC is aimed at possible microscopic residual tumor deposits. Consequently, the procedure is most appropriate after performing complete CRS.

There is currently no unified regimen or consensus on temperature, drug dosing, and optimal perfusion time of the HIPEC procedure. The scheme, dosage, and mode of the procedure were developed based on the results of the studies by Gonzalez et al. and Moldovan et al. [14, 15].

The combination of debulking surgery with HIPEC is performed annually on average among 15 patients per 1 million population [16].

Hyperthermic intraperitoneal chemotherapy is a promising option for patients with PC, which requires further study of clinical and oncological results and standardization of the approach.

The aim of the study was to investigate the intraoperative characteristics, postoperative complications, impact on the QOL, overall survival (OS) and disease-free survival (DFS) in patients with PC of different origin, who underwent HIPEC combined with CRS plus adjuvant chemotherapy (ACT) and to compare them with the standard of care $(C R S+A C T)$.

\section{Material and methods}

A total of 304 patients (77 male, 227 female) with PC of different origin, who underwent treatment and monitoring in the Center of Reconstructive and Renovative Medicine (University Clinic) of Odessa National Medical University during 2013-2021, were included in the single-institution retrospective non-randomized study.

Data were collected from our computerized surgical documentation system and archives of the institution and supplemented with data from our institution cancer follow-up program and the Ukrainian Cancer Registry. The study included only patients with fully collected information.

All patients undergoing treatment in the Center of Reconstructive and Renovative Medicine (University Clinic) of Odessa National Medical University have given their informed consent for participation in the research studies. This Center is one of two centers licensed to provide cancer care in the Odessa region and is the only one which provides surgical treatment in the advanced stages of oncological disease if it is possible. This analysis was approved by the local institutional review board, Odessa National Medical University Research Ethics Committee and was performed in accordance with the Helsinki Declaration of 1975, as revised in 1983.

Patients were divided into 2 groups:

- Group I - patients with PC who were treated with CRS + ACT - 247 patients,

- Group II - patients with PC, who were treated with CRS + HIPEC + ACT - 57 patients.

The stage of the disease was determined based on the International Classifications TNM $7^{\text {th }}$ and $8^{\text {th }}$ edition (according to the year of the initial diagnosis). Clinical, laboratory and instrumental examination of patients was performed according to the National Comprehensive Cancer Network, European Society for Medical Oncology, European Society of Gynecological Oncology and European Society of Surgical Oncology recommendations. The diagnosis of all patients was verified histologically and immunohistochemically.

The peritoneal cancer index $(\mathrm{PCl})$ was determined in the preoperative period using intrascopic methods (computed tomography with intravenous contrast and or magnetic resonance imaging using diffuse diffusion) and intraoperatively for all the patients [12]. The extent of CRS was determined depending on the spread of the tumor mass. Intraoperative characteristics were estimated in both groups: completeness of cytoreduction (CC), operative time, intraoperative blood loss), length of hospital stay (LOS), the incidence of early (up to 7 days after surgery) and late (8-30 days after surgery) postoperative complications grade II-IV according to Clavien-Dindo, postoperative mortality, return of bowel function (peristalsis and stool) after surgery. The analysis of the QOL of patients was performed using the international scales the Short Form36 (SF-36) and European Organization for Research and Treatment of Cancer Quality of Life Questionnaire Core 30 (EORTC QLQ-C30) version 3 before the surgery, before special treatment, 3 days after surgery, 20 days after surgery, before the fourth cycle of chemotherapy and 1 month after completion of chemotherapy. SF-36 is a non-specific questionnaire that reflects the well-being and satisfaction of those aspects of human life that are affected by health. EORTC QLQ-C30 version 3 is a specific questionnaire designed for patients with cancer. 
Median OS and median disease-free period were calculated for both groups in patients with different primary origins of PC.

The condition of patients in both groups was assessed by analyzing clinical, instrumental and laboratory parameters. After CRS + HIPEC on day 2, 5 and every 10 days during 1 month after surgery, ECG and blood tests were performed.

The patient selection criteria for CRS + HIPEC included ECOG status $0-1$, ASA I-III, age $\leq 65$ years, $P C I \leq 20$. The exclusion criterion for CRS + HIPEC was extraperitoneal metastatic spread. The hyperthermic intraperitoneal chemotherapy procedure for patients of Group II was performed using the RanD Performer HT system. An important advantage of the device is the ability to simulate the temperature of the perfusion solution during the procedure according to the data from temperature sensors. During the procedure, the flow rate of the perfusion solution, the volume of perfusion, and 6-channel control of the pressure of the perfusion solution in the system were monitored. All the patients of Group II underwent the same procedure of HIPEC. Perfusion of the abdominal cavity with a solution containing cytostatic drugs was performed at a temperature of $41^{\circ} \mathrm{C}$.

The total duration of the HIPEC procedure was 120 minutes, including a perfusion period of 90 minutes at a flow rate of 800-900 $\mathrm{ml} / \mathrm{min}$. Circulation of the chemotherapeutic agent was ensured by means of two pumps, a temperature cooler and a sterile closed circuit. The procedure was performed using the "closed abdomen" technique, which means infusion and evacuation of the perfusate is performed using 5 silicone tubes installed through separate incisions. Afterwards the abdominal wall was closed. After reaching an intra-abdominal temperature of $41^{\circ} \mathrm{C}$, cisplatin $\left(50 \mathrm{mg} / \mathrm{m}^{2}\right)$ and doxorubicin $\left(15 \mathrm{mg} / \mathrm{m}^{2}\right)$ were dissolved in 4-6 liters of perfusate (the volume of perfusate depended on the patient's weight $-60 \mathrm{ml} / \mathrm{kg}$ ). During the procedure, intra-abdominal temperature, body temperature, central venous pressure, heart rate, blood pressure, and urine output were monitored. Sodium thiosulfate was administered at the beginning of perfusion as an intravenous bolus $\left(7.5 \mathrm{~g} / \mathrm{m}^{2}\right)$ followed by continuous infusion $\left(25 \mathrm{~g} / \mathrm{m}^{2}\right)$ for 12 hours in order to reduce the nephrotoxic effect of chemotherapeutic drugs.

A comparison of groups of patients according to the criteria represented by numerical variables was performed using Student's t-test. Pearson's $\chi^{2}$ test was used to compare the frequencies of occurrence in the groups, and two-sided Fisher's test was used to determine the relationship. The log-rank test was used to compare OS and DFS.

\section{Results}

The average age of patients was $57.5 \pm 12.1$ years (Group I $-57.7 \pm 12$ years, Group $\|-56.7 \pm 12.4$ years; $p>0.05)$.

Patients with PC who were included in the study were diagnosed as follows:

- 126 with epithelial ovarian carcinoma (Group I - 91 patients, Group II - 35),

- 140 with colorectal adenocarcinoma (Group I - 131 patients, Group II -9),
- 30 with gastric adenocarcinoma (Group I - 24 patients, Group II-6),

- 8 with malignant peritoneal mesothelioma (Group I 1 patients, Group II - 7).

Alimentary status, family history, anemia and comorbidities (mixed comorbidities, cardiovascular diseases, respiratory diseases, urinary pathology, neuroendocrine pathology) of all patients in both groups were analyzed. Statistical homogeneity of groups $(p>0.05)$ was defined for each of the indicators. Between groups, there was no statistically significant difference in the mean $\mathrm{PCl}$, the extent of CRS or completeness of the cytoreduction $(p>0.05)$ (Tables 1, 2).

Intraoperative characteristics of patients are shown in Table 1. The average duration of the operation in Group II (CRS + HIPEC) was longer than in Group I (CRS + ACT) $\left(t_{\text {kr. }}<t_{\text {emp. }}[p<0.05]\right)$. Surgery duration in Group II (CRS + HIPEC + ACT) was increased due to the HIPEC procedure (120 minutes). There was no statistically significant difference in average intraoperative blood loss between groups $\left(t_{\text {kr. }}>t_{\text {emp. }}[p>0.05]\right)$. Intraoperative blood transfusion was performed in 97 (29.2\%) patients in Group I vs. 24 (42.1\%) in Group II ( $\left.p>0.05\left[\phi_{\text {emp. }}^{*}=0.155\right]\right)$, i.e. no statistically significant difference was obtained). However, the average volume of blood transfusion in patients who underwent it was significantly higher in Group II $\left(t_{k r}<t_{\text {emp. }}[p<0.05]\right)$.

The median LOS in Group II (CRS + HIPEC + ACT) was $12.37 \pm 5.1$ (4-27) days vs. 9.7 5.9 (4-32) days in Group I $(C R S+A C T)\left(t_{\text {kr. }}=2.58<t_{\text {emp. }}=3.4\right.$, so the difference is statistically significant $[p<0.01])$.

Clavien-Dindo grade II-IV postoperative complications occurred in 34\% (84) of patients (58 patients in the early postoperative period, 26 in the late postoperative period) in Group I (CRS + ACT) vs. 38.6\% (22 patients) (14 patients - in the early postoperative period, 8 - in the late postoperative period) in Group II. The list and the incidence of early and late postoperative complications in Group I (CRS $+\mathrm{ACT})$ and Group II (CRS + HIPEC + ACT) are presented in Tables 2 and 3.

The total rate of postoperative complications did not differ between groups ( $\left.p>0.05\left[\phi_{\text {emp. }}^{*}=0.653\right]\right)$. In the detailed analysis of early postoperative complications in patients of Group II (CRS + HIPEC + ACT) hyperthermia and acute renal failure occurred more often than in Group I $(\mathrm{CRS}+\mathrm{ACT})$ (for both complications $p<0.05)$, which was probably caused by the influence of the chemotherapeutic drugs and prolonged exposure of hyperthermic solutions in the abdominal cavity. The overall mortality was $2 \%$ (5 patients) in Group I (1 patient intraoperatively and 4 in the postoperative period) vs. 3.5\% (2 patients) in Group II (all in the postoperative period) (no statistically significant difference was obtained, $\left.p>0.05\left[\phi_{\text {emp. }}^{\star}=0.626\right]\right)$.

The median duration of recovery of digestive function was compared for the 2 groups:

- the peristalsis recovered after 1.9 (1-4) days in Group I vs. 3.5 (1-6) days in Group II (tkr.< temp., the difference is statistically significant $[p<0.01])$,

- defecation in Group I was observed after 3.3 (2-8) days vs. 5.1 (3-10) days in Group II (tkr.< temp., the difference is statistically significant $[p<0.01])$. 
Table 1. Intraoperative characteristics of patients in Group I (CRS + ACT) and Group II (CRS + HIPEC + ACT)

\begin{tabular}{|c|c|c|c|}
\hline & \multicolumn{2}{|c|}{ Group of patients } & \multirow[t]{2}{*}{$p$-value } \\
\hline & $\begin{array}{c}\text { I (CRS + ACT) } \\
n=247(\%)\end{array}$ & $\begin{array}{c}\text { II (CRS + HIPEC + ACT), } \\
n=57(\%)\end{array}$ & \\
\hline \multicolumn{4}{|l|}{ Intraoperative characteristic } \\
\hline $\mathrm{PCl}$ & $13.6+3.9$ & $11.2+5.2$ & $>0.05$ \\
\hline CC-O and CC-1 & $209(84.6)$ & $51(89.5)$ & $>0.05$ \\
\hline Laparotomy & $205(83)$ & $45(78.9)$ & $>0.05$ \\
\hline Laparoscopy & $42(17)$ & $12(21.1)$ & $>0.05$ \\
\hline Intraoperative blood loss (ml) & $510(100-2750)$ & $525(150-2300)$ & $>0.05$ \\
\hline Operation duration (min) & $187.3(74-364)$ & $310.1(215-372)$ & $<0.05$ \\
\hline Blood transfusion volume $(\mathrm{ml})$ & $710(440-2650)$ & $750(450-1480)$ & $<0.05$ \\
\hline Ostomy & $60(24.3)$ & $15(26.3)$ & $>0.05$ \\
\hline \multicolumn{4}{|l|}{ Surgical volume } \\
\hline Total/subtotal peritonectomy & $105(42.5)$ & $25(43.9)$ & $>0.05$ \\
\hline Intestinal anastomosis & $172(69.6)$ & $38(66.7)$ & $>0.05$ \\
\hline Appendectomy & $14(5.7)$ & $4(7)$ & $>0.05$ \\
\hline Hysterectomy and bilateral adnexectomy & $66(26.7)$ & $21(36.8)$ & $>0.05$ \\
\hline Adnexectomy & $6(2.4)$ & $5(8.8)$ & $>0.05$ \\
\hline Urinal anastomosis & $11(4.5)$ & $4(7)$ & $>0.05$ \\
\hline Total pelvic exenteration & $4(1.6)$ & $3(5.3)$ & $>0.05$ \\
\hline Anterior pelvic exenteration & $9(3.6)$ & $5(8.8)$ & $>0.05$ \\
\hline Posterior pelvic exenteration & $13(5.3)$ & $5(8.8)$ & $>0.05$ \\
\hline Urinary bladder resection & $24(9.7)$ & $8(14)$ & $>0.05$ \\
\hline Cholecystectomy & $18(7.3)$ & $6(10.5)$ & $>0.05$ \\
\hline Pancreatic resection & $9(3.6)$ & $1(1.8)$ & $>0.05$ \\
\hline Splenectomy & $42(17)$ & $10(17.5)$ & $>0.05$ \\
\hline Liver resection & $45(18.2)$ & $15(26.3)$ & $>0.05$ \\
\hline Diaphragm resection & 73 (29.6) & $11(19.3)$ & $>0.05$ \\
\hline
\end{tabular}

ACT - adjuvant chemotherapy, CRS - cytoreductive surgery, HIPEC - hyperthermic intraperitoneal chemotherapy

The use of HIPEC significantly contributed to the worse restoration of intestinal function in the postoperative period.

Patients in Group II (CRS + HIPEC + ACT) were evaluated for toxicity of chemotherapeutic treatment by analysis of clinical and laboratory parameters. After HIPEC on days 2 and 5 , as well as before each course of chemotherapy and between the third and sixth day of each course, ECG and hematological parameters were monitored, and biochemical blood tests were performed. No deaths associated with chemotherapy have been reported.

The data obtained in Group II (CRS + HIPEC + ACT) are similar to those in Group I (CRS + ACT). The most common manifestations of myelotoxicity were thrombocytopenia (in 38 (66.7\%) patients of Group II and 155 (62.75\%) patients of Group I) and leukopenia (in 5 (33.3\%) patients of Group II and 12 (27.91\%) patients of Group I).

Among the early (up to 7 days) non-hematological side effects of chemotherapy treatment, nausea and vomiting, and diarrhea were most common in the first 24 hours in 5 patients $(33.3 \%)$ and in $2(13.3 \%)$ patients during the first week from Group II compared with 13 (30.23\%) and 5 (11.63\%) patients, respectively, from Group I. Among the late delayed (7 days - 1 month) side effects of chemotherapeutic treatment, peripheral neuropathy was most often observed (in 8 (53.33\%) patients of Group II and 27 (62.79\%) patients of Group I). For late (from 1 month) side effects of chemotherapeutic treatment, the most characteristic was sexual dysfunction (in 9 (60\%) patients of Group II and 24 (55.81\%) patients of Group I).

Comparing the frequency of side effects of chemotherapeutic treatment in both groups $\chi^{2}=25.749, \chi^{2} \mathrm{kr}$. $=27.587$ ( $p=0.0577$ ), i.e. no statistically significant difference in the frequency of toxic effects of systemic chemotherapy of 3-4 degrees of severity was obtained.

In the study of QOL in patients with PC with SF-36, scores were calculated on 8 scales, which allowed us to assess the physical $(\mathrm{PH})$ and psychological $(\mathrm{MH})$ components of health. The range of parameters of each scale is $0-100$. Higher scores indicate a better QOL related to health. Questionnaires with SF-36 were conducted among both groups of patients with PC before special treatment, 3 days after surgery, 20 days after surgery, before the fourth cycle of chemotherapy and 1 month after completion of chemotherapy.

At the baseline (prior to special treatment), the mean $\mathrm{PH}$ was $47.44( \pm 9.4)$ in Group I and $47.21( \pm 9.8)$ in Group II, as shown in Figure 1. The mean levels of $\mathrm{MH}$ at baseline were $49.72( \pm 11.2)$ in Group I and 43.92 ( \pm 10.9$)$ in Group II (Fig. 2). 
Table 2. Early postoperative complications of patients in Group I (CRS + ACT) and Group II (CRS + HIPEC + ACT) grade II-IV according to Clavien-Dindo

\begin{tabular}{|c|c|c|c|}
\hline \multirow{2}{*}{$\begin{array}{l}\text { Early postoperative complications } \\
\text { (up to } 7 \text { days after operation) }\end{array}$} & \multicolumn{2}{|c|}{ Group of patients } & \multirow[t]{2}{*}{$p$-value } \\
\hline & $\begin{array}{c}\text { I (CRS + ACT) } \\
n=247(\%)\end{array}$ & $\begin{array}{c}\text { II (CRS + HIPEC + ACT) } \\
n=57(\%)\end{array}$ & \\
\hline Bowel perforation (acute ulcer) & $4(1.6)$ & $3(5.2)$ & $>0.05$ \\
\hline Coagulopathic bleeding & $4(1.6)$ & $3(5.2)$ & $>0.05$ \\
\hline Anastomotic leak & $10(4)$ & $5(8.8)$ & $>0.05$ \\
\hline Relaparotomy & $14(5.7)$ & $6(10.5)$ & $>0.05$ \\
\hline Eventration & $5(2)$ & $3(5.2)$ & $>0.05$ \\
\hline Ileus & $16(6.5)$ & $7(12.3)$ & $>0.05$ \\
\hline Infectious complications & $15(6.1)$ & $7(12.3)$ & $>0.05$ \\
\hline Hyperthermia & $15(8.9)$ & $10(15.7)$ & $<0.05$ \\
\hline Acute renal failure & $4(1.6)$ & $5(8.8)$ & $<0.05$ \\
\hline Acute liver failure & $8(3.2)$ & $3(5.2)$ & $>0.05$ \\
\hline Pneumothorax & $8(3.2)$ & $2(3.5)$ & $>0.05$ \\
\hline Pulmonary artery thromboembolism & $4(1.6)$ & $2(3.5)$ & $>0.05$ \\
\hline Postoperative pneumonia & $2(0.8)$ & $2(3.5)$ & $>0.05$ \\
\hline Deep vein thrombosis & $2(0.8)$ & $1(1.8)$ & $>0.05$ \\
\hline Myocardial infarction & $1(0.4)$ & 0 & $>0.05$ \\
\hline Cerebrovascular disorders & $2(0.8 \%)$ & $1(1.8)$ & $>0.05$ \\
\hline Anemia & $31(12.6)$ & $9(15.8)$ & $>0.05$ \\
\hline Perforation of gastric ulcer & $1(0.4)$ & $1(1.8)$ & $>0.05$ \\
\hline Bleeding from a gastric ulcer & $1(0.4)$ & 0 & $>0.05$ \\
\hline
\end{tabular}

Table 3. Late postoperative complications of patients in Group I (CRS + ACT) and Group II (CRS + HIPEC + ACT) grade II-IV according to Clavien-Dindo

\begin{tabular}{|c|c|c|c|}
\hline \multirow{2}{*}{$\begin{array}{l}\text { Late postoperative complications } \\
\text { (from } 8 \text { to } 30 \text { days after operation) }\end{array}$} & \multicolumn{2}{|c|}{ Group of patients } & \multirow[t]{2}{*}{$p$-value } \\
\hline & $\begin{array}{l}\text { I (CRS + ACT) } \\
n=247(\%)\end{array}$ & $\begin{array}{c}\text { II (CRS + HIPEC + ACT) } \\
n=57(\%)\end{array}$ & \\
\hline Colorectal anastomotic leak & $3(1.2)$ & $2(3.5)$ & $>0.05$ \\
\hline Relaparotomy & $7(2.8)$ & $5(8.8)$ & $>0.05$ \\
\hline Intestinal fistula & $6(2.4)$ & $5(5.2)$ & $>0.05$ \\
\hline Infectious complications & $9(3.6)$ & $5(5.2)$ & $>0.05$ \\
\hline Eventration & $5(2)$ & $3(5.2)$ & $>0.05$ \\
\hline Ventral hernia & $6(2.4)$ & $4(5.2)$ & $>0.05$ \\
\hline Anemia & $10(4)$ & $7(5.2)$ & $>0.05$ \\
\hline Gastric ulcer bleeding & 0 & $1(1.8)$ & $>0.05$ \\
\hline Deep vein thrombosis & $3(1.2)$ & $1(1.8)$ & $>0.05$ \\
\hline Hospital-acquired pneumonia & $6(2.4)$ & $3(5.2)$ & $>0.05$ \\
\hline Myocardial infarction & 0 & $1(1.8)$ & $>0.05$ \\
\hline Cerebrovascular disorders & $1(0.4)$ & 0 & $>0.05$ \\
\hline Acute renal failure & $3(1.2)$ & $3(5.2)$ & $>0.05$ \\
\hline Acute liver failure & $2(0.8)$ & $1(1.8)$ & $>0.05$ \\
\hline Abdominal lymphocyst & $4(1.6)$ & $1(1.8)$ & $>0.05$ \\
\hline
\end{tabular}

ACT - adjuvant chemotherapy, CRS - cytoreductive surgery, HIPEC - hyperthermic intraperitoneal chemotherapy 


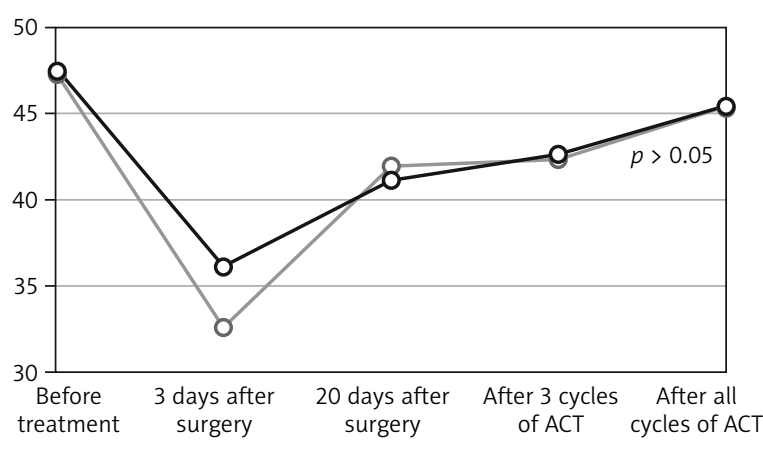

Fig. 1. The value of the physical component of the health of patients in Group I (CRS + ACT) - dark gray, II Group (CRS + HIPEC + ACT) light gray at each stage of treatment

ACT - adjuvant chemotherapy, CRS - cytoreductive surgery, HIPEC - hyperthermic intraperitoneal chemotherapy

There was no statistically significant difference in the parameters of $\mathrm{PH}$ and $\mathrm{MH}$ between groups in $\mathrm{PC}$ before the start of special treatment $(p>0.05)$.

Quality of life was assessed in the early ( $3^{\text {rd }}$ day) and late postoperative period ( $20^{\text {th }}$ day). In the early postoperative period, the average value of $\mathrm{PH}$ was $36.08( \pm 9.7)$ in Group I and 32.6 ( \pm 8.8 ) in Group II (Fig. 1); the mean value of MH was $38.89( \pm 9.5)$ and $39.15( \pm 10.1)$, respectively (Fig. 2). In the late postoperative period, the average value of $\mathrm{PH}$ was $41.1( \pm 9.3)$ in Group I and $41.93( \pm 10.4)$ in Group II (Fig. 1); the mean value of $\mathrm{MH}$ was $39.01( \pm 9.2)$ and 37.99 ( \pm 7.6$)$, respectively (Fig. 2).

During ACT (before the 4th course of ACT), the average value of PH was $42.61( \pm 10.3)$ in Group I and $42.33( \pm 9.1)$ in Group II (Fig. 1); the mean value of MH was $39.8( \pm 7.9)$ and 38.54 ( \pm 9.6$)$, respectively (Fig. 2). 1 month after the completion of ACT, the average value of PH was $45.44( \pm 10.8)$ in Group I and 45.3 \pm ) 9.7) in Group II (Fig. 1); the mean value of $\mathrm{MH}$ was $41.86( \pm 10.3)$ and $41.2( \pm 8.5)$, respectively (Fig. 2 ).

Pearson's $\chi^{2}$ test was used for a comparison of $\mathrm{PH}$ and $\mathrm{MH}$ parameters at all stages of treatment. When comparing the $\mathrm{PH}$ of both groups, an empirical $\chi^{2}$ of 0.36 and a critical $\chi^{2}$ of 9.49 were obtained $(p=0.98)$. When comparing $\mathrm{MH}$ of groups I and II, $\chi^{2}$ empirical 0.75 and $\chi^{2}$ critical 9.49 were obtained $(p=0.94)$.

These results demonstrate the absence of a significant difference in the parameters of $\mathrm{PH}$ and $\mathrm{MH}$ in patients with PC in both groups.

The presence of a malignant process, the localization of tumors and special treatment significantly affect the QOL of patients with PC. In particular, abdominal (gastrointestinal) symptoms are specific for patients with PC. Their presence can significantly reduce the QOL. Such symptoms include bloating, pain or cramps, dyspeptic symptoms and other digestive disorders. All the above can also occur as a result of special treatment of PC. Urological and gynecological symptoms are less common in patients with PC, but they can often be caused by the treatment. The most common are frequent urination and vaginal dryness. The most common side effects of PC chemotherapy are nausea and vomiting, poor appetite, alopecia, peripheral neuropathy, including numbness and weakness of the extremities, other sensory changes, skin symptoms, and muscle pain, which can

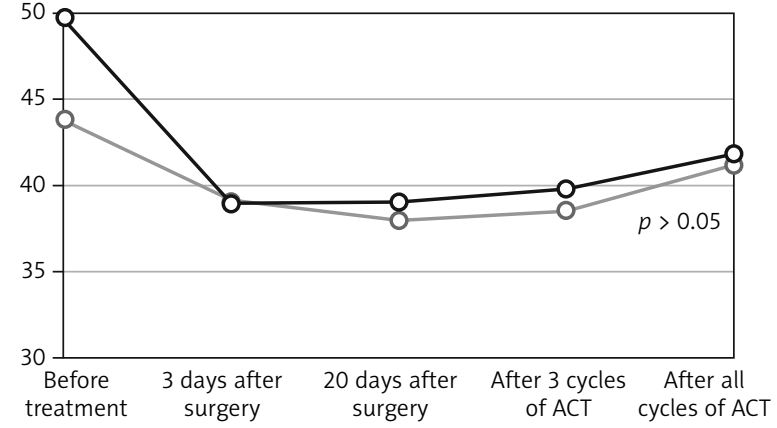

Fig. 2. The value of the psychological component of the health of patients in Group I (CRS + ACT) - dark gray, II Group (CRS + HIPEC $+\mathrm{ACT})$ - light gray at each stage of treatment

ACT - adjuvant chemotherapy, CRS - cytoreductive surgery, HIPEC - hyperther mic intraperitoneal chemotherapy

also be assessed with the EORTC QLQ-C30. Among women of reproductive age, both surgery and chemotherapy can cause early menopause. These patients have symptoms of menopause caused by hormonal depletion. Patients with PC are also characterized by negative dynamics on the scale of body image. Patients may feel less attractive, dissatisfied with their body or appearance. Quality of life of patients with PC can also be affected by negative dynamics on the scale of sexuality, which is determined by a decrease of interest in sex, sexual activity and pleasure. Therefore, to assess the QOL of patients with PC, the analysis of the presence and incidence of all these symptoms is important.

All data obtained by completing the EORTC QLQ-C30 were analyzed according to the recommendations of the EORTC group. Figures 3-7 show the values of all scales of the EORTC QLQ-C30 in patients of both groups at all stages of the investigation.

The indicators of functional and symptomatic scales, as well as the indicator of the overall QOL, were compared with Student's t-test in both groups. There was no statistically significant difference between the indicators of functional and symptomatic scales and the indicator of overall QOL before the start of special treatment $(p>0.05)$. In the early postoperative period increased scores of pain $\left(t_{k r}<t_{\text {emp. }}\right.$. $[p<0.05])$, nausea and vomiting $\left(t_{k r}<t_{\text {emp }}[p<0.05]\right)$, and constipation ( $\left.\mathrm{t}_{\mathrm{kr} .}<\mathrm{t}_{\mathrm{emp} .}[p<0.05]\right)$ were observed in Group II compared with Group I (statistically significant).

Comparing the parameters of functional scales, other symptom scales and overall QOL, the difference between the groups is statistically insignificant $\left(t_{\mathrm{kr}}<\mathrm{t}_{\mathrm{emp} .}[p<0.05]\right)$. Subsequently, comparing both groups of PC patients with the Student's t-test, there is no significant difference between functional and symptomatic scales and overall QOL in the late postoperative period, after the fourth course of ACT and 1 month after ACT $\left(t_{k r}>t_{\text {emp. }}[p<0.05]\right)$.

The median OS of patients with colorectal cancer in Group I was 23.7 months (range, 0-71) vs. 29.2 months (range, 5-64) in Group II (no significant difference using log rank test, $p=0.106)$.

The median OS of patients with ovarian cancer in Group I was 27.4 months (range, 0-78) vs. 36.1 months (range 0-76) in Group II (significant difference using log rank test, $p=0.013)$. 

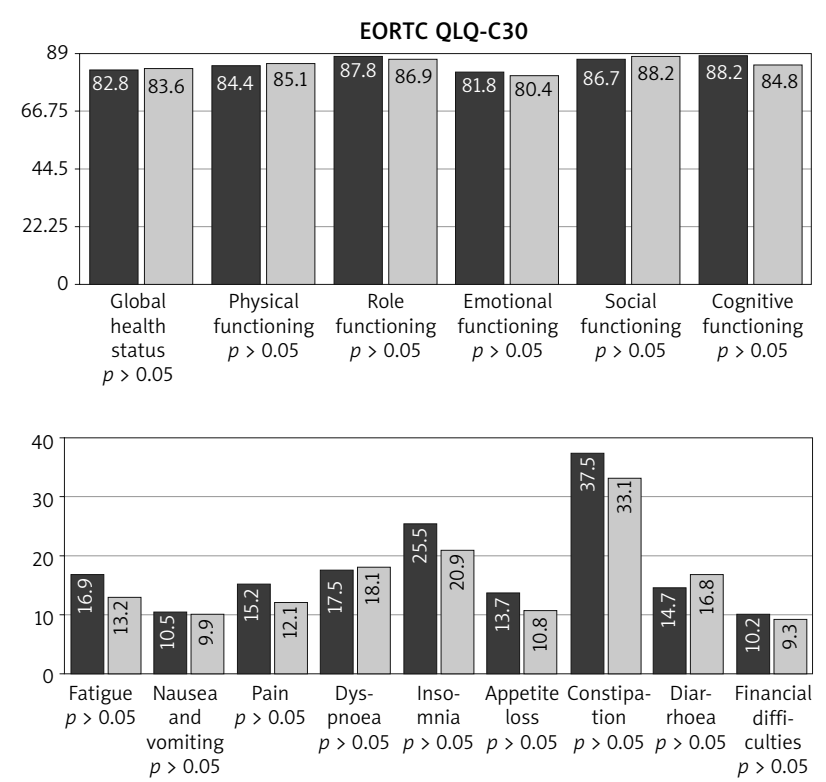

Fig. 3. Values of the EORTC QLQ-C30 scales of patients in Group I $(C R S+A C T)$ - dark gray, Group II $(C R S+$ HIPEC + ACT $)$ - light gray at each stage of treatment, before special treatment

ACT - adjuvant chemotherapy, CRS - cytoreductive surgery, EORTC QLQ-C3O - European Organization for Research and Treatment of Cancer Quality of Life Questionnaire Core 30, HIPEC - hyperthermic intraperitoneal chemotherapy

The median OS of patients with gastric cancer in Group I was 12.5 months (range, 0-41) and was not reached in Group II.

The median DFS of patients with colorectal cancer in Group I was 11.8 months (range, 0-29) and 14.1 months (range, 3-25) in Group II (no significant difference using log rank test, $p=0.33$ ).

EORTC QLQ-C30
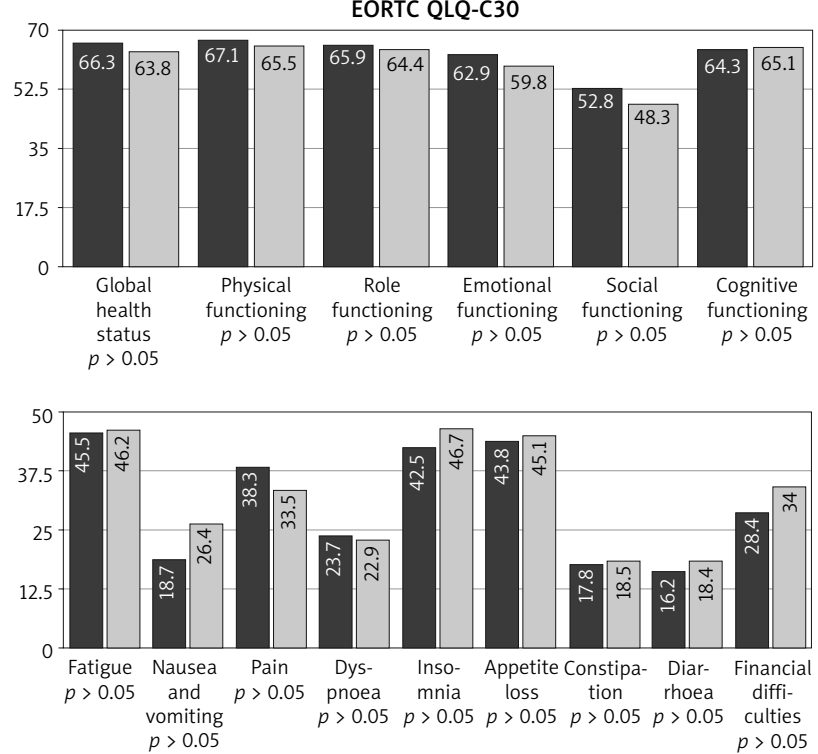

Fig. 5. Values of the EORTC QLQ-C30 scales of patients in Group I $(C R S+A C T)$ - dark gray, Group II (CRS + HIPEC + ACT $)$ - light gray at each stage of treatment, 20 days after surgery

ACT - adjuvant chemotherapy, CRS - cytoreductive surgery, EORTC QLQ-C30 - European Organization for Research and Treatment of Cancer Quality of Life Questionnaire Core 30, HIPEC - hyperthermic intraperitoneal chemotherapy
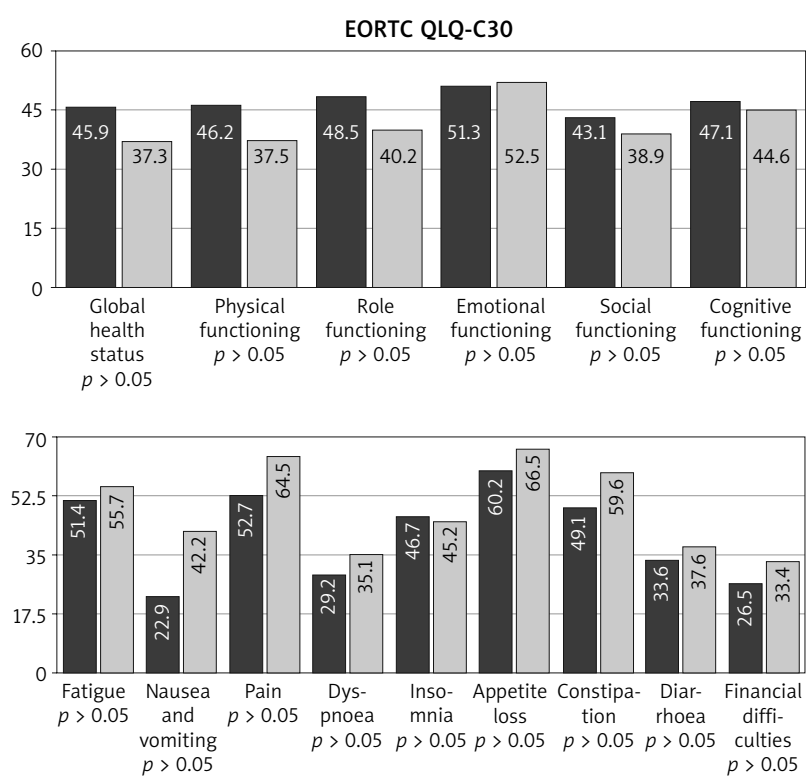

Fig. 4. Values of the EORTC QLQ-C30 scales of patients in Group I $(C R S+A C T)$ - dark gray, Group II (CRS + HIPEC + ACT $)$ - light gray at each stage of treatment, 3 days after surgery

ACT - adjuvant chemotherapy, CRS - cytoreductive surgery, EORTC QLQ-C3O - European Organization for Research and Treatment of Cancer Quality of Life Questionnaire Core 30, HIPEC - hyperthermic intraperitoneal chemotherapy

The median DFS of patients with ovarian cancer in Group I was 16.4 months (range, 0-38) and 21.1 months (range 0-35) in Group II (significant difference using log rank test, $p=0.005$ ).

The median DFS of patients with gastric cancer in Group I was 8.1 months (range, 0-22) and 9.2 months (range, 0-19) in Group II (no significant difference using log rank test, $p=0.479$ ).
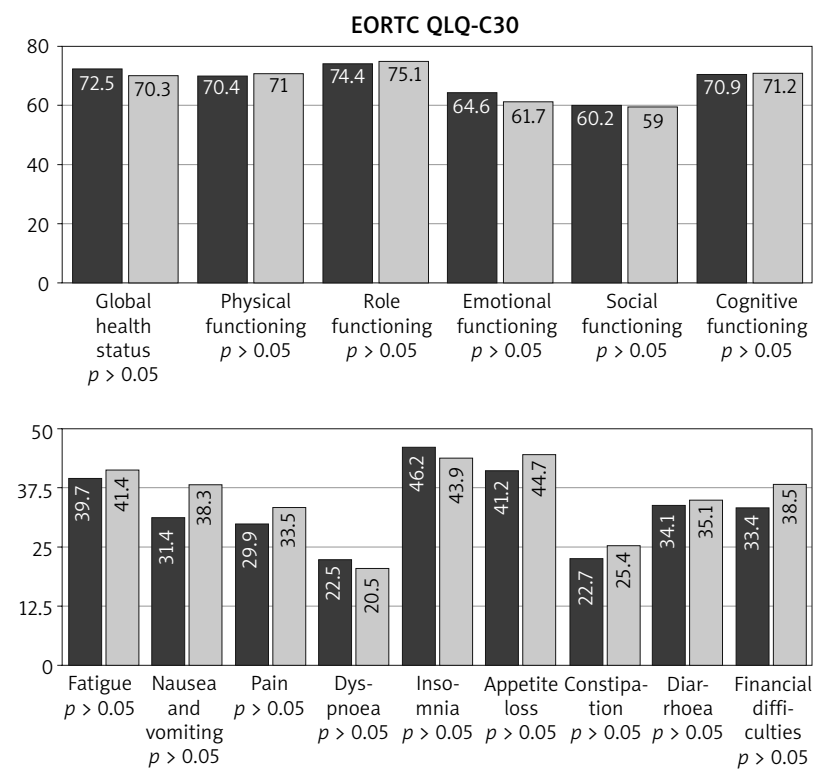

Fig. 6. Values of the EORTC QLQ-C30 scales of patients in Group I $(C R S+A C T)$ - dark gray, Group II (CRS + HIPEC + ACT $)$ - light gray at each stage of treatment, after 3 courses of ACT

ACT - adjuvant chemotherapy, CRS - cytoreductive surgery, EORTC QLQ-C30 - European Organization for Research and Treatment of Cancer Quality of Life Questionnaire Core 30, HIPEC - hyperthermic intraperitoneal chemotherapy 


\section{Discussion}

Hyperthermic intraperitoneal chemotherapy involves the intraperitoneal delivery of cytostatic drugs during surgery under hyperthermic conditions. Its pathogenetic effectiveness was described previously. Investigating its safety and impact on the clinical results and QOL of patients precedes the assessment of the final effectiveness, in the case of PC - the oncological results. Hyperthermic intraperitoneal chemotherapy is an optional method of treatment and in most of the guidelines it is recommended for use as a part of clinical trials and research.

$85.5 \%$ of patients included in this study $(84.6 \%$ in Group I and $89.5 \%$ in Group II) underwent CC-0 or CC-1 CRS. Despite the worse clinical results and QOL due to the aggressive surgical approach, a direct positive relationship between oncological results and the completeness of CRS is proved for patients with PC of different primary origins [17]. So the main goal in the cytoreductive surgery of patients with PC is to achieve complete cytoreduction. In this case the use of HIPEC is pathogenetically grounded.

In this study the use of HIPEC in Group II led to increased surgery duration and an increased volume of blood transfusion compared to Group I. Other intraoperative characteristics did not differ between groups. In the early postoperative period, the incidence rates of hyperthermia and acute renal failure in Group II (CRS + HIPEC + ACT) was statistically significantly higher than in Group I (CRS + ACT), although the number of total postoperative complications did not differ. In the investigation of Friedrich et al. the side effects and postoperative complications of III and IV grades of severity in patients with HIPEC occurred in $44.2 \%$ and $7 \%$ respectively [18]. Zhou et al. observed rates of $47.7 \%$ for postoperative complications and $25.6 \%$ for high-grade complications in HIPEC + CRS patients [19]. Cardi et al. noted the postoperative complications rate of 35.3-53\% in HIPEC patients depending on their nutritional status [20]. A lower rate of grade III-IV postoperative complications was recorded by Polom et al. (18.7) [21]. To sum up, the rate of postoperative complications after CRS + HIPEC varies greatly in different investigations depending on the ECOG status of the patient, $\mathrm{PCl}$, performed surgery volume, HIPEC regimens, etc.

In this study, the median LOS in the HIPEC group was longer compared with the CRS + ACT group. In other studies, in different countries and populations LOS of the HIPEC patients was 15.4 days, 17 days, 12 days, 8.9 days, respectively [19, 22-24]. The bowel function return (peristalsis and stool) was slower in the HIPEC group compared with CRS + ACT $(p<0.01)$. These indicators could be modified by the rationalization of the perioperative approach and implementation of Enhanced Recovery After Surgery [25].

Concerning the QOL of patients who underwent HIPEC, significant deterioration was observed (using EORTC QLQ-C30) due to increased levels of pain, nausea and vomiting, stool retention, and gastrointestinal symptoms in the early postoperative period. In the subsequent stages of special treatment and after its completion, no significant deterioration in the QOL was observed among patients who underwent HIPEC compared to the control group. No difference in QLQ between groups was obtained using the
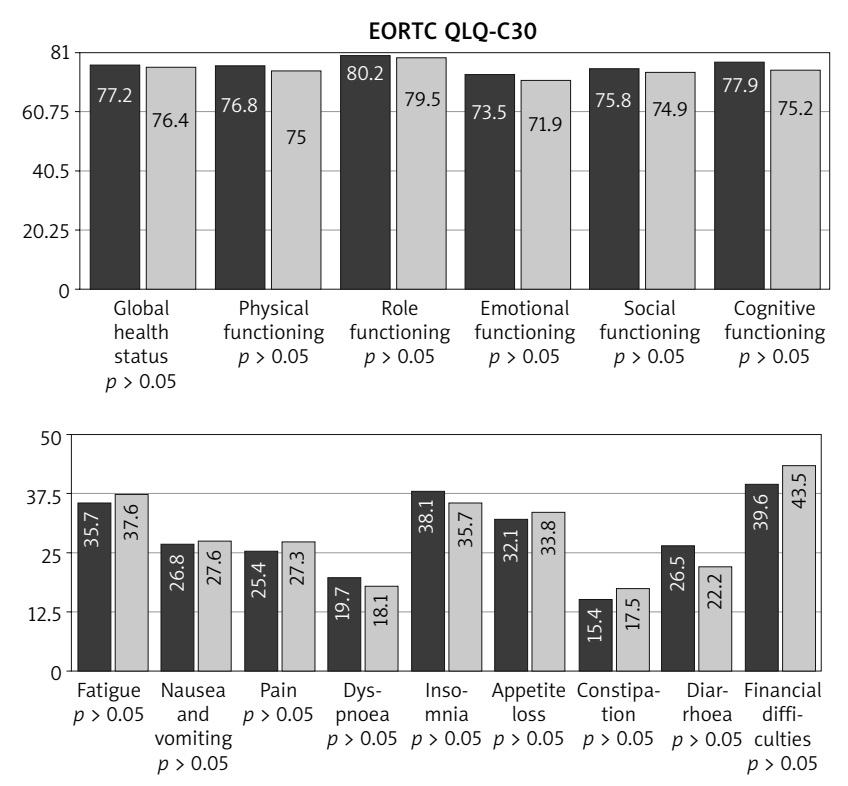

Fig. 7. Values of the EORTC QLQ-C30 scales of patients in Group I $(C R S+A C T)$ - dark gray, Group II (CRS + HIPEC + ACT $)$ - light gray at each stage of treatment, one month after completion of ACT ACT - adjuvant chemotherapy, CRS - cytoreductive surgery, EORTC QLQ-C30 - European Organization for Research and Treatment of Cancer Quality of Life Questionnaire Core 30, HIPEC - hyperthermic intraperitoneal chemotherapy

SF-36 questionnaire. In a study by Rybin et al., QOL (using SF-36) in patients who underwent CRS + HIPEC compared with CRS alone did not differ significantly 6 months after treatment completion [24]. In the investigation of Dodson et al. a significant reduction in QOL in patients after HIPEC using SF-36 was obtained, although this change was completely offset within 6 months [26]. According to the analysis of the literature and our results, it is recommended to use the specific cancer EORTC QLQ-C30. Due to the multimodal assessment this questionnaire can more accurately determine each parameter of QOL to correct pathological conditions and to modify treatment. However, Lustosa et al. [27] and Koole et al. [28] found no significant reduction in QOL of the ovarian cancer patients using the EORTC questionnaire in the group of HIPEC and interval CRS after neo ACT (carboplatin AUC 6 and paclitaxel 175 mg/m²) compared to the control group.

Overall survival and DFS vary greatly depending on the primary origin of $\mathrm{PC}, \mathrm{PCl}, \mathrm{CC}$ and the time of $\mathrm{PC}$ occurrence (synchronous or metachronous). In this study, OS and DFS were analyzed regarding the primary origin of PC. Significant increases of OS and DFS were obtained in the HIPEC group in patients with ovarian cancer.

Due to obtained clinical outcomes and assessed QOL of patients treated with standard methods and those who received HIPEC, the following stages of the study should be performed to assess early and late oncological outcomes (recurrence and OS rates) in the stratified groups regarding the primary origin and the time of PC development (synchronous and metachronous).

\section{Conclusions}

Hyperthermic intraperitoneal chemotherapy technique has demonstrated its clinical safety in the treatment of PC. 
The average volume of intraoperative blood loss and the incidence of all postoperative complications in patients who underwent HIPEC and CRS did not statistically significantly exceed similar parameters in the group without HIPEC. However, the rates of hyperthermia and acute renal failure in the early postoperative period were higher in the HIPEC group. In patients with PC who underwent the HIPEC procedure there was a deterioration in QOL according to specific EORTC QLQ-C30 in the early postoperative period compared with those who underwent only CRS. At the subsequent stages of treatment, there was no significant difference between the functional, symptomatic scales and the overall QOL of patients who underwent HIPEC compared with patients who underwent CRS alone. Assessment of the QOL of patients who underwent HIPEC with SF-36 showed no significant difference in the $\mathrm{PH}$ and $\mathrm{MH}$ components of health at all stages of treatment compared with those who underwent CRS alone, either. Thus, HIPEC simultaneously with CRS does not notably impair the clinical outcomes of patients and can be recommended for the treatment of PC. Also, the use of specific cancer questionnaires is recommended for a more objective assessment of the QOL of patients with PC. Overall survival and DFS of patients with ovarian cancer who underwent HIPEC were significantly better compared with CRS + ACT alone. The oncological outcomes of HIPEC are still under investigation for colorectal and gastric cancer and other primary localizations of PC.

\section{The authors declare no conflict of interest.}

\section{References}

1. Desai JP, Moustarah F. Peritoneal metastasis. Available from: https://www.ncbi.nlm.nih.gov/books/NBK541114/.

2. Zakharenko AA, Zaitcev DA, Belyaev MA, et al. Modern strategy in treatment of peritoneal carcinomatosis. Sci Notes PU 2018; 24: 7-12.

3. Sushkov OI, Achkasov SI. Peritoneal colorectal carcinomatosis. Approaches to treatment (review). Coloproctol 2016; 4: 69-79.

4. Dong XD. Right sided colon cancer and peritoneal carcinomatosis. Ann Laparosc Endosc Surg 2019; 4: 72-72.

5. Yarema R, Ohorchak M, Hyrya P. Gastric cancer with peritoneal metastases: efficiency of standard treatment methods. World J Gastrointest Oncol 2020; 12: 569-581.

6. Wang Z, Chen Jq, Liu J, et al. Issues on peritoneal metastasis of gastric cancer: an update. World J Surg Onc 2019; 17: 215.

7. Van Baal JOAM, van Noorden CJF, Nieuwland R, et al. Development of peritoneal carcinomatosis in epithelial ovarian cancer: a review. J Histochem Cytochem 2018; 66: 67-83.

8. Akhtar M, Haider A, Rashid S, Al-Nabet ADMH. Paget's "seed and soil" theory of cancer metastasis. Adv Anatom Pathol 2019; 26: 69-74.

9. Jaaback K, Johnson N, Lawrie TA. Intraperitoneal chemotherapy for the initial management of primary epithelial ovarian cancer. Cochrane Database Syst Rev 2016; 1: CD005340.

10. Oei AL, Vriend LE, Krawczyk PM, et al. Targeting therapy-resistant cancer stem cells by hyperthermia. Int J Hyperthermia 2017; 33: 419-427.

11. Sharma A, Özayral S, Caserto JS, et al. Increased uptake of doxorubicin by cells undergoing heat stress does not explain its synergistic cytotoxicity with hyperthermia. Int J Hyperthermia 2019; 36: 712-720.

12. Bhatt $A$, Glehen $\mathrm{O}$. The role of cytoreductive surgery and hyperthermic intraperitoneal chemotherapy (HIPEC) in ovarian cancer: a review. Indian J Surg Oncol 2016; 7: 188-197.
13. Vergote I, Harter P, Chiva L. Is there a role for intraperitoneal chemotherapy, including HIPEC, in the management of ovarian cancer? J Clin Oncol 2019; 37: 2420-2423.

14. Gonzalez L, Steiner, M, Vasquez J, et al. Cytoreductive surgery and hyperthermic intraperitoneal chemotherapy for the treatment of advanced epithelial ovarian carcinoma: upfront therapy, at first recurrence, or later? Eur J Surg Oncol 2013; 39: 1109-1115.

15. Moldovan B, Moldovan D, Capilna A. The St. Constantin Hospital's initial experience with CRS-HIPEC. J Transl Med Res 2015; 20: 246-252.

16. Verwaal VJ, Rau B, Jamali F, et al. Registries on peritoneal surface malignancies throughout the world, their use and their options. Int J Hyperthermia 2017; 33: 528-533.

17. Chiva L, Lapuente F, Castellanos T, et al. What should we expect after a complete cytoreduction at the time of interval or primary debulking surgery in advanced ovarian cancer? Ann Surg Oncol 2015; 23: 1666-1673.

18. Friedrich M, Zinn W, Kolnsberg L, et al. Hyperthermic intraperitoneal chemotherapy (HIPEC) for ovarian cancer: evaluation of side effects in a single institution cohort. Anticancer Res 2020; 40: 1481-1486.

19. Zhou S, Feng Q, Zhang J, et al. High-grade postoperative complications affect survival outcomes of patients with colorectal cancer peritoneal metastases treated with cytoreductive surgery and hyperthermic intraperitoneal chemotherapy. BMC Cancer 2021; 21: 41.

20. Cardi M, Sibio S, di Marzo F, et al. Prognostic factors influencing infectious complications after cytoreductive surgery and HIPEC: results from a tertiary referral center. Gastroenterol Res Pract 2019; 2019: 2824073.

21. Polom K, Roviello G, Generali D, et al. Cytoreductive surgery and hyperthermic intraperitoneal chemotherapy for treatment of ovarian cancer. Int J Hyperthermia 2016; 32: 298-310.

22. Chen WC, Huang HJ, Yang LY, et al. Hyperthermic intraperitoneal chemotherapy for recurrent epithelial ovarian cancer. Biomed J 2021; S2319-4170(21)00137-2.

23. Gamboa AC, Lee RM, Turgeon MK, et al. Implications of postoperative complications for survival after cytoreductive surgery and HIPEC: a multi-institutional analysis of the US HIPEC collaborative. Ann Surg Oncol 2020; 27: 4980-4995.

24. Rybin Al, Maksymovskyj VY, Pyrogov VV, et al. Comparative study of quality of life, adverse effects after cytoreduction and HIPEC in stage IIIA-IIIC ovarian cancer. J Edu Health Sport 2018; 8: 412-417.

25. Tkachenko O, Chetverikov S, Bondar O, Maksymovskyi V, Chetverikov $M$, Chetverikova-Ovchynnyk $V$. Implementation of the enhanced recovery after surgery protocol for patients with peritoneal carcinomatosis undergoing cytoreductive surgery and hyperthermic intraperitoneal chemoperfusion. Contemp Oncol (Pozn) 2021; 25: 133-139.

26. Dodson RM, McQuellon RP, Mogal HD, et al. Quality-of-life evaluation after cytoreductive surgery with hyperthermic intraperitoneal chemotherapy. Ann Surg Oncol 2016; 23 (Suppl 5): 772-783.

27. Lustosa RJ, Batista TP, Carneiro VC, et al. Quality of life in a phase 2 trial of short-course hyperthermic intraperitoneal chemotherapy (HIPEC) at interval debulking surgery for high tumor burden ovarian cancer. Rev Col Bras Cir 2020; 47: e20202534.

28. Koole SN, Kieffer JM, K Sikorska, et al. Health-related quality of life after interval cytoreductive surgery with or without hyperthermic intraperitoneal chemotherapy (HIPEC) in patients with stage III ovarian cancer. Eur J Surg Oncol 2019; S0748-7983(19)30444-5.

\section{Address for correspondence}

\section{Valeriia Volodymyrivna Chetverikova-Ovchynnyk}

Odessa National Medical University

16/61 Generala Petrova St.

65078, Odessa, Ukraine

e-mail: ovchinnik.val@yahoo.com

Submitted: 29.08 .2021

Accepted: 14.11 .2021 